\title{
L'insertion des usines de traitement d'eau en milieu urbain - contraintes et résultats
}

\author{
Didier Haegel (*), François Blondeau (**) \\ (*) OTV Méditerranée, 700, avenue Nicolas Fabri de Peiresc - ZI de Toulon Est - 83088 Toulon Cedex \\ (**) SPACE International, 14, rue de Mantes - 92711 Colombes Cedex
}

La réalisation d'usines de traitement d'eau, et notamment de stations d'épuration en milieu urbain, est parfois une nécessité. Elle implique le respect de contraintes spécifiques : l'emploi de procédés compacts, la mise en auvre de fonctions complémentaires, une sécurité et une exploitabilité renforcée... Des réalisations spectaculaires montrent que l'on peut associer ces contraintes à une protection optimale de l'environnement. Ce sont les usines de: Marseille, Monaco, Antibes, St Cyr-sur-Mer, la Ciotat, Col de Villefranche, St Tropez, Embrun.

Le 12 octobre 1977 était publié en France le décret 77-1141 rendant obligatoire la réalisation d'une étude d'impact pour les ouvrages d'épuration des eaux usées des collectivités locales. Cette action n'était que la traduction du souci de nos concitoyens de se voir protégés contre les nuisances de tous ordres. Mais les usines de traitement d'eau sont des ouvrages relativement importants dont l'implantation doit, en premier lieu tenir compte de contraintes liées à la topographie et à l'architecture du réseau. Ceci est particulièrement vrai pour les stations d'épuration des villes littorales où, par nature, la zone d'urbanisation dense est aussi celle où se concentrent les collecteurs vers le point de rejet à l'exutoire.

Les concepteurs des usines d'eau ont été amenés à réaliser des ouvrages situés soit en ville soit au bord de mer, dans des zones très sensibles où leur intégration implique la mise en œuvre de solutions spécifiques tant au niveau des procédés qu'à celui de leur adaptation aux sites d'accueil.

La réussite, en l'espèce implique le respect des concepts suivants :

- réduction de l'impact visuel et intégration architecturale,

- prévention des nuisances olfactives et sonores en limitant l'interface "station-atmosphère ",

— confinement des installations.

Ainsi se dessinent les caractéristiques d'une usine compacte porteuse du label « Nuisance zéro » qui outre sa fonction première de traitement de l'eau, respecte aussi tous les aspects de l'environnement.

\section{Les contraintes des usines d'épuration " nuisance zéro »}

Des procédés " compacts "

Pour réduire l'interface entre l'usine et son voisinage, les concepteurs font appel à des procédés de traitement compacts et, par conséquent, à haute charge volumique. C'est ainsi que l'on a vu se développer au cours des dernières années la technologie de la décantation lamellaire et des procédés d'épuration biologique par culture fixée sur lit immergé.

La première par la mise en place de radiers intermédiaires entre la surface et le radier assure en même temps : la diminution du temps nécessaire aux particules pour atteindre la surface de dépôt et l'accroissement de l'aire de dépôt par mètre carré de surface libre. A efficacité égale, un décanteur lamellaire occupe une surface 5 à 10 fois plus faible que celle d'un ouvrage conventionnel. Ce rendement 
peut encore être accru par la technique des flocs lestés comme dans le procédé ACTIFLO.

Les filtres immergés combinent, quant à eux, en un seul ouvrage, deux fonctions : celle de réacteur biologique, à celle de clarificateur de filtration mécanique. Le procédé BIOCARBONE qui est le premier biofiltre immergé à avoir été développé en France occupe avec ses annexes une surface de $1000 \mathrm{~m}^{2}$ pour 100000 habitants, contre les 3000 et $3500 \mathrm{~m}^{2}$ que mobiliserait l'application d'un procédé conventionnel par boues activées avec cuve d'aération et clarification finale, pour l'élimination de la pollution carbonée. Le procédé BIOSTYR qui est le dernier développement en matière de biofiltration immergée autorise, grâce à la technologie du médium filtrant plus léger que l'eau, des vitesses et des charges encore accrues et, par conséquent, constitue une avancée substantielle dans le domaine des procédés compacts d'épuration biologique.

Au plan du traitement des boues, la recherche de la compacité conduit à la généralisation de filières associant une déshydratation mécanique des boues fraîches à une stabilisation chimique des boues déshydratées. La centrifugation apparaît comme une technologie bien adaptée grâce à l'encombrement réduit des équipements. Son association à une stabilisation chimique par apport de chaux permet d'obtenir aisément des résidus dont la siccité dépasse $30 \%$ de matières sèches, valeur compatible avec une évacuation et une dévolution finale économiques.

\section{Des fonctions complémentaires}

La prévention des nuisances, et, notamment l'élimination des risques d'émission d'odeurs, implique un confinement quasi total des ouvrages.

En effet, les ouvrages de traitement et notamment les stations d'épuration, sont le siège d'émissions de gaz qu'il est impératif de collecter et traiter avant rejet à l'atmosphère.

Compte tenu de leur origine et des caractéristiques physico-chimiques qui en résultent, les eaux usées sont un milieu réducteur et présentent un terrain très favorable au déroulement de réactions de putréfaction en phase acide, sources d'odeurs particulièrement puissantes et insupportables. Certains des gaz émis (H2S, CH4) sont, au-delà d'un certain seuil de concentration, toxiques et dangereux pour les personnes. Parallèlement, et nous y reviendrons, leur présence participe à des phénomènes de corrosion contre lesquels il faut conduire des actions de prévention.

L'ensemble de ces phénomènes serait accru par le confinement si l'on ne menait pas une action compensatrice de dilution des gaz à l'intérieur du volume fermé par le biais d'une ventilation très poussée. Les taux de renouvellement varient en fonction des risques encourus dans les différentes zones fonctionnelles; ils se situent dans une plage de 3 à 10 renouvellements par heure avec une moyenne de 5 à 6 .

La mise en œuvre d'une épuration des gaz de ventilation est le corollaire du confinement. Si dans la majorité des cas cette épuration est réalisée par des procédés physicochimiques basés sur des réactions d'oxydo-réduction en milieu aqueux, il existe également des situations où une épuration biologique des gaz par filtration sur un lit bactérien adapté peut s'avérer être une solution appropriée.

La ventilation participe également à la protection des équipements électro-mécaniques contre la corrosion qui ne manquerait pas de se produire en raison d'une trop grande humidité, et de la présence de certains gaz agressifs (H2S).

Le confinement des ouvrages et la prévention contre la corrosion incitent à la mise en œuvre de matériaux résistants à la corrosion (acier inoxydable, alliages d'aluminium) ou mieux, incorrodables par nature (matières plastiques).

\section{Une sécurité et une exploitabilité renforcées}

Le confinement accroît la gravité des conséquences d'accident. Des mesures renforcées de détection des risques doivent être appliquées (risques d'explosion, d'incendie, de toxicité, d'inondation...). Les équipements de sécurité doivent donc être fiabilisés (redondances) et secourus (groupe électrogène, source autonome...).

Enfin, la station d'épuration confinée dans un volume aussi restreint que possible doit être exploitable aisément. Ainsi, chaque équipement doit être accessible pour les opérations d'entretien courant. Ils doivent être démontables et les dispositions prises pour assurer leur transport jusqu'au site d'intervention.

L'éclairage (le plus souvent artificiel), doit être suffisamment puissant et éviter les zones d'ombres.

Une station d'épuration fermée comporte des axes de pénétration et les moyens de levage et de manutention (monorails, ponts roulants, tracteurs...) adaptés aux équipements fonctionnels qu'elle comporte.

Pour le confort du personnel, et éviter des problèmes liés à la claustration, la prise en compte d'aspects ergonomiques (éclairages variés, peintures, décoration florale...) ne doit pas être négligée.

\section{Des structures nouvelles}

Les usines de traitement à ciel ouvert font appel très largement aux structures simples dont la réalisation est économique.

Au sein des stations totalement fermées, la recherche de l'économie de surface l'emporte sur l'économie propre à la structure. Les ouvrages sont donc essentiellement parallélépipédiques, et par conséquent plus onéreux.

La réalisation des stations d'épuration de la nouvelle génération incite à l'application de méthodes d'exécution où la préfabrication d'éléments ou de modules peut tenir une place importante.

Enfin, n'oublions pas que la station « nuisance zéro » est couverte et que souvent cette couverture sert de support à un aménagement complémentaire : stade, terre plein, parc, etc... ce qui n'est pas sans incidence sur la structure.

OTV, précurseur dans ce domaine des ouvrages intégrés en sites urbains, vous présente donc quelques exemplaires d'insertion réussis, choisis dans la région Provence Alpes Côte d'Azur. 


\section{STATION D'ÉPURATION DE MARSEILLE (13)}

\section{Caractéristiques techniques}

a) Données entrée

* Population desservie $1700000 \mathrm{hbts}$

* Débit journalier $312000 \mathrm{~m}^{3} / \mathrm{j}$

* Débit de pointe $7,40 \mathrm{~m}^{3} / \mathrm{s}$

* Charge en MES $687 \mathrm{t} / \mathrm{j}$

* Concentration en MES $218 \mathrm{mg} / \mathrm{l}$

b) Objectif d'épuration

* Concentration en MES $<50 \mathrm{mg} / \mathrm{l}$

\section{Procédés retenus}

* Prétraitements.

* Prédécantation 14 ouvrages

* Décantation lamellaire "CLARIFLO ".. 18 ouvrages "La plus grande concentration au monde de décanteurs lamellaires ",

\section{Caractéristiques d'intégration}

* Surface occupée $25000 \mathrm{~m}^{2}$

* Entièrement enterrée, recouverte par un stade (en pleine ville à proximité du stade vélodrome).

* Débit de ventilation $400000 \mathrm{~m}^{3} / \mathrm{h}$

* Année de mise en service 1987

\section{STATION D'ÉPURATION DE LA} PRINCIPAUTÉ DE MONACO (MC)

\section{Caractéristiques techniques}

a) Données entrée

* Population desservie 100000 hbts

* Débit journalier $31000 \mathrm{~m}^{3} / \mathrm{j}$

* Débit de pointe $1800 \mathrm{~m}^{3} / \mathrm{h}$

* Charges en DBO $6300 \mathrm{~kg} / \mathrm{j}$

* Charges en DCO $12500 \mathrm{~kg} / \mathrm{j}$

* Charges en MES $3800 \mathrm{~kg} / \mathrm{j}$

b) Objectif d'épuration

* MES $20 \mathrm{mg} / 1$

* DBO $20 \mathrm{mg} / 1$

\section{Procédés retenus}

* Prétraitements : dans un tunnel sous le rocher de Monaco.
* Décantation lamellaire " CLARIFLO » avec traitement physico-chimique 2 ouvrages

* Filtration biologique par procédé BIOCARBONE. 11 filtres

* Traitement des boues par une filière comprenant :

- épaississement,

- centrifugeuse,

- séchage thermique à $95 \%$ de siccité,

ou • four incinération dans le jour d'ordures ménagères situé dans un bâtiment jouxtant la station d'épuration.

* Désodorisation par lavage chimique. Débit de ventilation : $68000 \mathrm{~m}^{3} / \mathrm{h}$

\section{Caractéristiques d'intégration}

Située dans un immeuble de 10 niveaux abritant des bureaux, de petites industries, un stand de tir, implanté en pleine ville dans le quartier de Fontvieille.

Le traitement des eaux occupe une surface de $2900 \mathrm{~m}^{2}$ hors-tout sur 2 niveaux de sous-sol, le point bas étant situé à $-12 \mathrm{~m}$ sous la mer.

Le traitement des boues et la désodorisation occupent 5 niveaux sur une partie de l'immeuble, la projection au sol étant de $350 \mathrm{~m}^{2}$.

Année de mise en service 1990

\section{STATION D'ÉPURATION DE LA CIOTAT (13)}

\section{Caractéristiques techniques}

a) Données entrée

* Population desservie 50000 hbts

* Débit journalier $10000 \mathrm{~m}^{3} / \mathrm{j}$

* Débit de pointe $750 \mathrm{~m}^{3} / \mathrm{h}$

* Charge en DBO $2700 \mathrm{~kg} / \mathrm{j}$

* Charge en MES $3100 \mathrm{~kg} / \mathrm{j}$

b) Objectif d'épuration

Niveau c - soit $90 \%$ d'élimination des MEST.

\section{Procédés retenus}

* Prétraitements.

* Traitement physico-chimique avec coagulation prolongée et décantation lamellaire "CLARIFLO ".

* Traitement des boues par épaississement et centrifugation.

* Désodorisation par lavage chimique.

L'air désodorisé étant rejetté dans le tirant d'air de l'émissaire de rejet en mer. 


\section{Caractéristiques d'intégration}

* Surface occupée $1500 \mathrm{~m}^{2}$

* Située en pleine ville entre 2 boulevards à l'arrière des chantiers navals.

* L'originalité de cette usine consiste en sa qualité architecturale. Non dissimulée, elle a été conçue comme un ouvrage de structuration du quartier grâce à son escalier monumental, ses terrasses paysagées, son habillage de façade et la qualité de ses matériaux.

* Année de mise en service 1990

\section{STATION D'ÉPURATION D'ANTIBES-JUAN-LES-PINS (06)}

1. Caractéristiques techniques

a) Données entrée

* Population desservie $172000 \mathrm{hbts}$

* Débit journalier $40500 \mathrm{~m}^{3} / \mathrm{j}$

* Débit de pointe $3375 \mathrm{~m}^{3} / \mathrm{h}$

* Charge en DBO $10500 \mathrm{~kg} / \mathrm{j}$

* Charge en DCO $22000 \mathrm{~kg} / \mathrm{j}$

* Charge en MEST $10500 \mathrm{~kg} / \mathrm{j}$

b) Objectif d'épuration

Niveau e.

\section{Procédés retenus}

* Prétraitements.

* Traitement physico-chimique avec décantation lamellaire "CLARIFLO » 4 ouvrages

* Filtration biologique «BIOCARBONE » ..... 10 filtres

* Emplacement prévu pour désinfection à l'ozone ultérieurement.

* Traitement des boues :

- épaississement,

- centrifugation,

- chaulage à sec des boues déshydratées.

* Ventilation: débit $100000 \mathrm{~m}^{3} / \mathrm{h}$.

* Désodorisation par lavage chimique en 2 files parallèles de 3 tours.

\section{Caractéristiques d'intégration}

* La station est implantée sur un terrain situé au pied de la colline de La Garoupe (Cap d'Antibes) et à l'arrière du port de la Salis dans une zone urbanisée de type résidentiel.

* De type semi-enterrée, la station occupe une superficie de $6500 \mathrm{~m}^{2}$. La toiture et l'environnement immédiat ont fait l'objet d'un traitement paysagé particulièrement soigné.

* Année de mise en service 1990

\section{STATION D'ÉPURATION} DE ST-CYR-SUR-MER (83)

\section{Caractéristiques techniques}

a) Données entrée

* Population desservie 23000 hbts

* Débit journalier $4600 \mathrm{~m}^{3} / \mathrm{j}$

* Débit de pointe $400 \mathrm{~m}^{3} / \mathrm{s}$

* Charge en DBO $1250 \mathrm{~kg} / \mathrm{j}$

* Charge en MES $1500 \mathrm{~kg} / \mathrm{j}$

b) Objectif d'épuration

Niveau b soit $80 \%$ d'élimination des matières en suspension.

\section{Procédés retenus}

* Prétraitements.

* Traitement physico-chimique avec décantation lamellaire " CLARIFLO ".

* Traitement des boues par :

- épaississement,

- filtration sur bande.

* Désodorisation sur lit de tourbe.

* Débit de ventilation : $20000 \mathrm{~m}^{3} / \mathrm{h}$

\section{Caractéristiques d'intégration}

La station d'épuration est située dans une pinède à proximité d'une zone résidentielle. Entièrement fermée, elle occupe une surface d'environ $900 \mathrm{~m}^{2}$.

Année de mise en service 1988

\section{STATION D’ÉPURATION DE ST-TROPEZ (83)}

\section{Caractéristiques techniques}

a) Données entrée

* Population desservie 32000 hbts

* Débit journalier $6400 \mathrm{~m}^{3} / \mathrm{j}$

* Débit de pointe $600 \mathrm{~m}^{3} / \mathrm{h}$

* Charge en DBO $1700 \mathrm{~kg} / \mathrm{j}$

* Charge en MES $2200 \mathrm{~kg} / \mathrm{j}$

b) Objectif d'épuration Niveau e.

\section{Procédés retenus}

* Prétraitements.

* Traitement physico-chimique avec décantation lamellaire "CLARIFLO ".

* Filtration biologique par procédé BIOCARBONE. 


\section{USINES DE TRAITEMENT D'EAU EN MILIEU URBAIN}

* Traitement des boues par épaississement et centrifugation.

* Désodorisation par lavage chimique.

\section{Caractéristiques d'intégration}

* La station d'épuration de St-Tropez est implantée au pied de la citadelle à proximité du cimetière des Graniers.

* Entièrement souterraine, seule sa façade en pierre sèche apparaît, en harmonie avec les remparts de la Citadelle.

* Année de mise en service du biologique 1991

\section{STATION D'ÉPURATION D'EMBRUN (05)}

\section{Caractéristiques techniques}

a) Données entrée

* Population desservie 35000 hbts

* Débit journalier $4800 \mathrm{~m}^{3} / \mathrm{j}$

* Débit de pointe $400 \mathrm{~m}^{3} / \mathrm{s}$

* Charge en DBO $1577 \mathrm{~kg} / \mathrm{j}$

* Charge en DCO $3470 \mathrm{~kg} / \mathrm{j}$

* Charge en MES $2044 \mathrm{~kg} / \mathrm{j}$

b) Objectif d'épuration

Niveau e.

\section{Procédés retenus}

* Prétraitements.

* Traitement physico-chimique avec :

- coagulation prolongée,

- décantation lamellaire "CLARIFLO " couplée à l'épaississement des boues.

* Filtration biologique par procédé «BIOCARBONE ».

* Centrifugation des boues.

* Désodorisation par lavage chimique.

\section{Caractéristiques d'intégration}

* Non dissimulée, mais néanmoins implantée dans un site très fréquenté, la station d'épuration d'Embrun grâce à une architecture très originale mettant en œuvre plans inclinés, mouvements paysagers et bâtiments bas, se fond à l'environnement.

* Année de mise en service 1991

\section{USINE DE TRAITEMENT D'EAU POTABLE DU COL DE VILLEFRANCHE (06)}

\section{Caractéristiques techniques}

a) Données entrée

* Populations desservies : Villes du Syndicat du Canal de la Moyenne Corniche (Villefranche, Eze, Beaulieu, St-Jean Cap Ferrat, Menton, etc...).

* Débit journalier $43200 \mathrm{~m}^{3} / \mathrm{j}$

* Eau brute Canal de la Vésubie

\section{Procédés retenus}

* Décantation par clarifloculation (Procédé « CLYCLO FLOC $n)$.

* Filtration bicouche.

* Désinfection à l'ozone.

* Remise à l'équilibre.

\section{Caractéristiques d'intégration}

Cette usine, bien que relativement conventionnelle dans sa conception est exceptionnelle par le fait qu'elle est implantée dans un site classé parmi les plus prestigieux de la Côte d'Azur, surplombant tout à la fois la Baie des Anges et la rade de Villefranche. Le pari architectural y a été réussi de belle manière.

Année de mise en service 1989

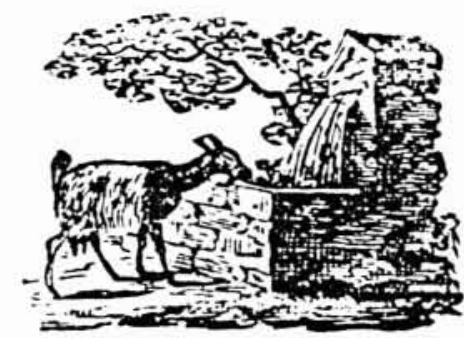

